\title{
Principles for Managing Patients with Spinal Ailments in the Coronavirus Disease 2019 Era: What Do We Know So Far? An Evidence-Based, Narrative Review
}

\author{
Vibhu Krishnan Viswanathan ${ }^{1}$, Surabhi Subramanian ${ }^{2}$, Arthi K. Rao ${ }^{3}$ \\ ${ }^{I}$ Department of Spine Surgery, Ganga Hospital, Coimbatore, India \\ ${ }^{2}$ Department of Radiodiagnosis, Ganga Hospital, Coimbatore, India \\ ${ }^{3}$ Division of Clinical Microbiology, Department of Laboratory Services, MIOT International, Chennai, India
}

\begin{abstract}
The coronavirus disease 2019 (COVID-19) pandemic has significantly affected all specialty practices in medicine, including the field of spinal surgery. Spinal surgery is unique in that the procedures include not only fully elective and fully emergent interventions, but also involve a separate group of semi-emergent surgeries, where delayed intervention may lead to permanent neurological deficits. Here, we present an evidence-based review on the impact of the COVID-19 pandemic on spinal surgery and our current knowledge about this issue. We conducted a thorough search of the PubMed, Medline, and Google Scholar databases using the keywords, "COVID-19," "COVID-19 impact on spine surgery," "coronavirus impact on spine surgery," "COVID-19 impact on neurosurgery," "coronavirus impact on neurosurgery," "COVID-19 impact on spine surgeons," and "coronavirus impact on spine surgeons" on May 6, 2020. A total of 8,322 articles were identified in the initial search. Articles that were duplicated, those that did not pertain to COVID-19 or spine surgeries, those with details not pertaining to the current topic of interest, and those published in languages other than English were excluded from our analyses. After complete screening, six articles were included in this review. During the previous few weeks, the COVID pandemic has significantly influenced all major aspects of spine surgery across the world. Outpatient care has been gradually shifted from physical visits to tele-health and online consultations. General recommendations have favored the conservative approach over surgeries, although no patient should be deprived of standard care owing to concerns about COVID. The general principles followed by spine surgeons should include early detection of COVID symptomatology; triaging of patients based on underlying spinal pathology; prescription of appropriate investigations to confirm the COVID status; isolation, as needed; selection of optimal management method as per the guidelines; adherence to best intraoperative practices; and ensuring protective measures for non-infected patients, family members, fellow heath care providers, and themselves against the disease.
\end{abstract}

Keywords: COVID-19; Surgical spinal procedure; Operative; Pandemics; Spine

Received May 18, 2020; Accepted May 21, 2020

Corresponding author: Vibhu Krishnan Viswanathan

Department of Spine Surgery, Ganga Hospital, Coimbatore, Tamil Nadu, India

Tel: +91-7358622093, E-mail: drvibu007@gmail.com 


\section{Introduction}

The term "pandemic" is derived from the Greek words "pan" meaning all and "demos" meaning people [1]. Hippocrates defined "crisis" (Green word "krisis") as the "turning point of a disease when important changes occur, indicating death or recovery" [2]. Today, the world is going through a crisis secondary to the pandemic caused by severe acute respiratory syndrome coronavirus 2 (SARSCoV-2) or coronavirus disease 2019 (COVID-19) that originated in Wuhan, the capital of Hubei, China $[3,4]$.

The full impact of the crisis is yet to be determined; nevertheless, its influence on specialty medicine practices across the world is well-recognized [5]. The field of spinal surgery is unique, considering that it not only constitutes an intersection of the clinical practice of orthopedic and neurosurgeons, but also caters to the following three different patient categories: those who require surgery emergently, those who are candidates for non-emergent interventions and another intermediate category, including patients who definitely benefit from early (although not emergent) surgery and are disadvantaged by a significant delay. We present an evidence-based review on the impact of COVID-19 on spinal surgery and our current knowledge on this subject.

\section{Methods}

We conducted a thorough search on the PubMed, Med- line, and Google Scholar databases using the keywords, "COVID-19," "COVID-19 impact on spine surgery," "coronavirus impact on spine surgery," "COVID-19 impact on neurosurgery," "coronavirus impact on neurosurgery," "COVID-19 impact on spine surgeons," and "coronavirus impact on spine surgeons" on April 26, 2020. We did not employ any specific selection criteria (Preferred Reporting Items for Systematic Reviews and Meta-Analyses [PRISMA] or Methodological Index for Non-Randomized Studies [MINORS] scoring criteria) to include articles in this narrative review.

\section{Results}

A total of 8,322 articles that were identified in the initial search were subjected to elaborate screening. Articles that were duplicated, those that did not pertain to COVID-19 or spine surgeries, articles with details not pertaining to the current topic of interest, and those published in a language other than English were excluded (Fig. 1). Finally, six articles were included. To our knowledge, no case series or original reports have been published on this subject. All the articles published so far include recommendations, editorials, guidelines, technical specifications, and observations on the current situation.

\section{Discussion}

In December 2019, a series of unexplained, viral pneumo-
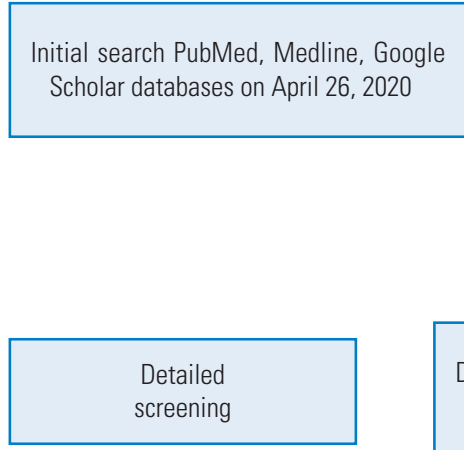

Final

inclusion

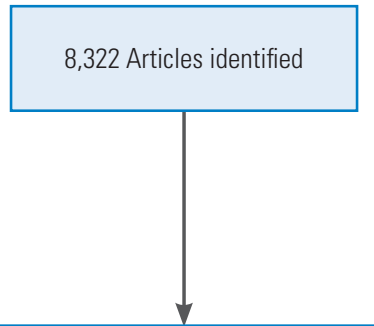

Duplicate articles, articles not pertaining to COVID-19 or spine surgeries, articles with details not pertaining to the current topic of interest, and nonEnglish language studies were excluded

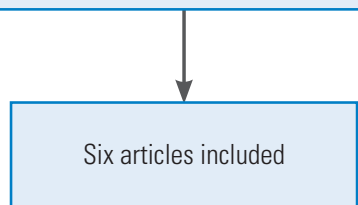

Fig. 1. Flowchart depicting the strategy employed for including and screening articles for the narrative review. COVID-19, coronavirus disease 2019 
nia cases were detected in the city of Wuhan, China. The causative organism was identified as a novel coronavirus on January 9, 2020. The virus was officially designated as World Health Organization (WHO) SARS-CoV-2 on February 11,2020 , and the disease was declared a pandemic on March 11, 2020 [6].

Although all major disasters in the history of the world have led to heavy losses on multiple fronts, they have also paved way for extra-ordinary breakthroughs, especially in the field of medicine. In fact, the field of neurosurgery itself gained prominence after World War-I. With the support of recent, substantial advancements in the fields of technology and engineering, the new lessons learnt from the current pandemic situation may prove instrumental in helping humanity take huge strides in ameliorating various facets of the health care delivery system. Thus, we planned this review to consolidate the present knowledge regarding the influence of the COVID-19 pandemic on spine surgeries at a time when the pandemic is reaching its peak in several countries of the world. We have comprehensively discussed various aspects of the influence exerted by this pandemic on spine health, independently from patient's, health care worker and hospital's perspectives. Thus far, few studies have addressed this issue.

\section{Pathophysiology of COVID-19}

The most essential proteins that enable the entry of the coronavirus into human cells are angiotensin converting enzyme- 2 and trans-membrane serine protease- 2 [4]. The former is a receptor protein, while the latter is a protease enzyme that allows the entry of the virus into cells. These proteins are abundantly present in goblet and ciliated epithelial cells of the nasal mucosa, cornea, and intestinal epithelium. The main sources of infection are confirmed patients, asymptomatic patients, and incubation-period patients. The most common routes of transmission include respiratory droplets and direct contact; aerosol and vertical propagation are other possible transmission methods. The incubation period is $2-14$ days $[7,8]$. The overall mortality rates vary from $0.2 \%$ to $15 \%$, depending on the age group and co-morbidities $[2,8]$.

The common symptoms include fever, sore throat, dry cough, fatigue, myalgia, nasal congestion, dyspnea, and diarrhea [9]. In case of critical patients, disease progression is generally rapid and can lead to loss of internal homeostasis, sepsis, acute respiratory distress syndrome, and multi-organ dysfunction syndrome [10]. In $20 \%$ of the patients, cardiac tissue damage has been observed secondary to the invasion of pericytes in cardiac blood vessels, myocytes, and fibroblasts by the viruses [5]. Although Chen et al. [11] showed that elderly patients with chronic comorbidities developed more severe disease, more recent reports have provided conflicting evidence of cytokine storms (raised interleukin [IL]-1, IL-10, and tumor necrosis factor alpha) in immuno-competent, young individuals directly contributing to more severe symptoms [12]. Thus, a high index of suspicion needs to be applied in the outpatient clinics, irrespective of the age of the patient being examined. On April 29, 2020 at 20:00 Greenwich mean time, there were 3,059,642 COVID-positive patients across 213 countries, and 211,028 deaths had already been reported [13].

\section{Outpatient clinics}

In most countries, outpatient spinal, orthopedic, or neurosurgical care is now being provided via tele-health (online) consultations to mitigate disease transmission [5]. Globally, elective outpatient appointments and visits have been significantly restricted. Research has shown that elderly patients with poor mobility and multiple comorbidities are highly susceptible to COVID-19 and have poor prognosis [14]. Most spine patients with degenerative pathologies belong to this category of patient population.

Patients with suspected or confirmed infection or disease are first required to visit fever clinics; thereafter, the spine surgeon may examine the patient, using full personal protective equipment (PPE). The same principles need to be followed in the emergency department. Segregation of outpatient clinics for fever and non-fever patients (with separate waiting areas) has also been recommended. The consultation room must be provided with disinfectant equipment, including quick-drying hand disinfectant and sodium hypochlorite solution. Indoor ventilation in the consultation room must be adequately enhanced, and all equipment must be carefully cleaned and disinfected after each consultation. In general, it is important to be judicious in the prescription of investigations (especially imaging studies like plain radiographs, computed tomography $[\mathrm{CT}]$, and magnetic resonance imaging) on outpatient basis, ensuring the prevention of any inadvertent crowding at these facilities and consequent cross-infections. 


\section{Personnel protection in outpatient clinics and emer- gency department}

The principle of social distancing must be followed in clinics with the maximum possible diligence to limit the spread of the infection to the medical staff, the patients, and people accompanying the patients. The duty rosters of the staff should be carefully organized, and the number of team members should be strictly limited [15]. All members of the medical staff should ideally wear proper attire, including goggles, disposable caps, National Institute for Occupational Safety and Health-approved (N95) masks, protective clothing, disposable gloves, and shoe covers [16]. The number of accompanying individuals should be strictly restricted to one, and the staff should strictly maintain a gap of $1 \mathrm{~m}$ from the patient or the accompanying individuals unless necessary for physical examination or some intervention. After contact with any patient, the gloves should be changed, and hands should be washed and disinfected [5]. All the instruments and medical equipment need to be thoroughly disinfected.

\section{Diagnostic modalities for COVID-19}

The diagnosis of COVID is based on clinical symptoms, contact history, travel history, laboratory investigations, and imaging studies [17]. The current laboratory tests to detect the virus include the following: (1) detection of the nucleic acid of SARS-CoV-2 with reverse transcriptionpolymerase chain reaction (RT-PCR), (2) genomic sequencing highly homologous to SARS-CoV-2, and (3) serological testing to detect specific immunoglobulin $G$ and immunoglobulin $\mathrm{M}$ antibodies to SARS-CoV-2. The total leukocyte count may be normal or low, while Creactive protein level and erythrocyte sedimentation rate are increased. Plain radiography and CT of the chest are important imaging modalities that can identify the typical pattern of viral pneumonia. Air bronchogram, multiple ground glass opacities, pulmonary consolidation, pleural thickening, and effusion are common imaging findings of the chest.

No specific guidelines have been proposed for ideal COVID-19 testing before spinal surgeries. However, guidelines proposed for orthopedic and neurosurgical procedures may be relevant for all spine patients $[18,19]$. Nasopharyngeal and oropharyngeal swabs are collected routinely; however, lower respiratory tract samples, such as sputum and bronchoalveolar lavage, can also be sent for testing [20]. RT-PCR has been recommended in preoperative situations owing to its high sensitivity and high negative predictive value. Limited data exist in support of routine antibody testing in preoperative situations because the kits that are used demonstrate variable accuracy and the virus exhibits very high genetic variability. Nextgeneration sequencing, CRISPR/Cas, and other molecular techniques may also be used as adjuncts, wherever available.

\section{Goals for managing patients with spinal ailments during the COVID-19 era}

Every spine surgeon should be aware of the major goals for managing patients with spinal disease during this crucial period; these goals include but are not restricted to the following: (1) ensuring availability of standard care for all patients, irrespective of their COVID-status and current situation; (2) early diagnosis: identification of the COVID status of patients (at the appropriate time), wherever necessary with appropriate investigations; (3) early support and management of COVID patients with spinal ailments: management of COVID positive individuals by an appropriate team of specialists and timely intervention in critical patients; (4) isolation of the infected and noninfected individuals as per the current recommendations; (5) prevention of cross-infections among patients, accompanying individuals, and health care providers as well as any spread of infection to the community; (6) minimizing the possibility of infection development in patients during their hospital stay; (7) minimizing any contamination of the hospital rooms, ORs, and equipment to ensure adequate decontamination via disinfection and sterilization after the exposure; (8) properly triaging the patients with spinal ailments based on the pathology and recommending a suitable line of treatment; (9) preventing, minimizing, or postponing spinal surgeries wherever feasible; prevention of spinal surgeries that have a longer surgical duration, such as deformity surgeries, reducing the scope of surgery, and using minimally invasive procedures whenever possible; (10) ensuring adequate protection of all OR personnel, anesthesia staff, and surgical teams, with strict adherence to all the recommended protocols; (11) avoidance of any excessive strain on the hospital resources during such difficult times; (12) ensuring timely discharge of patients from the hospital facility and ensuring that the 
patient is COVID-free, whenever possible, before returning to the community; and (13) being a good team player during such "uncertain hours" and be willing to contribute to the welfare for patient and community in any possible way.

\section{Patient categories based on COVID status and spinal pathology}

All patients being admitted to the hospital should be subjected to thorough evaluation for infection by an expert panel. All patients, irrespective of the clinical symptoms, should also undergo laboratory testing (preferably nucleic acid detection with RT-PCR) to identify the COVID status at the time of admission. During the COVID era, any patient admitted to the hospital needs to be classified into one of the following categories: (1) undocumented COVID-status without any suspected infection, (2) undocumented COVID-status with suspected infection, (3) documented COVID negative status, (4) documented COVID-positive status with asymptomatic infection, and (5) documented COVID-positive status with symptomatic disease $[5,21]$. All non-infected patients should be kept in a separate ward. Laboratory test (nucleic acid detection) and chest CT should be re-examined in patients with elective surgeries 3 days before the day of the operation; and surgery is performed after the diagnosis is excluded [5]. Patients with suspected COVID infection require single isolation, while all patients with symptomatic or asymptomatic infections need intensive isolation [5].

\section{COVID-19 and elective spine surgeries}

The consensus among surgeons is to avoid elective surgeries during the active period of the pandemic. However, to our knowledge, there are no SARS-CoV-2-specific risk stratification systems in place for elective spine or orthopedic surgeries. Patients who have risk factors, such as age $>75$ years, body mass index $>40 \mathrm{~kg} / \mathrm{m}^{2}$, diabetes mellitus, chronic obstructive pulmonary disease, chronic heart disease including hypertension, active cancer, and ongoing chemotherapy or any other immune-compromised states should be discouraged from undergoing elective spinal surgeries during this phase. Elective orthopedic surgeries must be delayed in patients with active COVID until they have been declared as "recovered" as per the local guidelines. The Centers for Disease Control and
Prevention has proposed guidelines to define recovery that includes 2 weeks of quarantine and absence of fever or other symptoms [22]. The current discharge policy for a known COVID-19 patient includes the normalization of chest radiograph findings and viral clearance on two specimens collected 24 hours apart [23]. It is critical to provide detailed education to all patients undergoing elective procedures regarding the protocols that have been followed to minimize the transmission of disease to themselves, other patients, family members, and health personnel. The American College of Surgeons, American Society of Anesthesiologists, Association of Perioperative Registered Nurses, and American Hospital Association have purported the roadmap for resuming elective surgeries in the coming days [24]. They have recommended that a sustained reduction in new COVID cases in the particular geographic area for at least 2 weeks is essential before considering full resumption of elective surgeries after authorization from the appropriate local, state, and national health authorities [25].

\section{Triaging spine surgeries based on pathology}

As previously mentioned, although a significant proportion of spine surgeries are "theoretically elective", in many spine patients, a considerable delay in care can result in less predictable and poorer outcome after surgery. Moreover, in certain conditions, a delay in neurological decompression can actually worsen the neurological deterioration and cause irreversible harm to patients. In the present scenario, there is significant uncertainty regarding the normalization of the scheduling of elective surgeries, potentially affecting overall patient care. Both, elective and emergency surgeries in symptomatic and asymptomatic COVID-19 positive patients can increase their mortality risk related to the use of general anesthesia. Thus, non-operative treatment should always be preferred over surgical treatment in these situations. Nevertheless, each COVID-19 patient deserves an excellent standard of care of treatment and should not be refused treatment for the fear of the infection [15].

Recently, the Rothman Institute has issued guidelines on the triaging of spine patients. The level 1 spine pathologies include cervical or thoracic myelopathies, spinal tumors, acute spinal trauma, epidural abscess, severe nerve root compression, and Cauda Equina syndrome. They classified patients with acute or sub-acute lumbar disc 
prolapse with intractable pain, cervical radiculopathy with intractable pain, acute postoperative implant failures, and lumbar adjacent segment disease as level 2 pathologies. The level 3 spinal pathologies included compression fractures without neurodeficit, odontoid fractures in elderly patients, lumbar stenosis, degenerative scoliosis, proximal junctional kyphosis, and axial low back pain. While the recommendation for level 1 pathologies is to proceed with urgent surgery at the hospital location, level 2 pathologies are managed surgically at the ambulatory surgical center or in a hospital facility with low COVID-19 census. In level 3 pathologies, surgery is preferably deferred or conservative management is seriously considered. In general, across specialties, non-urgent surgeries that require patient hospitalization for $>23$ hours have been postponed or cancelled. Alternative options, such as selective nerve root blocks may be considered in patients with severe radiculopathy.

\section{Spinal trauma and emergency situations}

In the present COVID pandemic situation, the management of spinal trauma and emergencies should differ from that for elective spinal surgeries. Sornsa-Ard et al. [26] recently published a flowchart for the management of spinal trauma patients wherein they classified patients into the stable, borderline, unstable, and extremis (or coma) categories. In the latter three groups, complete patient history may not be available; further, intubation and ventilation are often necessary. Such patients should be assessed initially in the designated isolation areas. The surgeons should use full PPE and hold discussions with local COVID teams about the arrangement of the required separate ward or separate intensive care units. Emergency surgeries may be performed with full PPE, preferably based on the principles of damage control orthopedics initially [27]. In such situations, COVID testing with PCR can be performed after the surgery and further management may be based on the COVID test results. Definitive surgical management in these patients can be performed after determining the final COVID status.

For patients who are stable and conscious, they recommended applying screening criteria that consisted of the following questions: (1) exposure to COVID-19, (2) risk of contact with COVID-19-positive subject or having tested positive for the viral infection, and (3) symptoms of cough and body temperature $\geq 37.5^{\circ}$ in WHO phase 2 or body temperature $\geq 37.3^{\circ}$ in WHO phase 3 regions. If a patient has any of the aforementioned factors, he should be provided a surgical face mask, examined in a designated isolation area by a surgical team wearing PPE, with infection control measures taken to perform emergency surgical procedures without waiting for the COVID results [28]. In patients who do not have any of these symptoms or with low exposure risk to COVID, surgical face masks may be provided to avoid any nosocomial infection, and standard surgical procedure may be performed under standard infection control precautions.

\section{Protection of healthcare and operating room personnel}

With more COVID-19-positive patients being admitted to hospitals, the risk of infection to the spinal surgeons and other operating room (OR) staff increases significantly. Detailed policies regarding the PPE need to be developed by each hospital [29]. Although the practice implemented by China, where the medical staff were tested every day should ideally be adopted world-wide; the limited availability of test kits and prevalence of false-negative reports (up to $30 \%$ ) greatly limit the testing ability [2]. Any health worker with suspicious symptoms needs to be isolated for timely medical management. When treating COVID-19positive patients or those with unknown COVID status, the anesthesiologists should wear N95 masks preferably with a powered air purifying respirator and a hood covering the head and neck areas during intubation [15]. All intubations in these patients must be performed outside the OR; if performed inside the OR, all the OR personnel should leave the room for at least 30 minutes after intubation to avoid exposure of surgical team to the aerosolizing effect on viruses during the process of respiratory management. Although ORs with negative pressure ventilation facility are preferred, they are not mandatory [15].

\section{Healthy intraoperative practices}

Although ORs are highly sterilized zones, and all the instruments and equipment are usually disinfected well, such an atmosphere does not always guarantee full safety. A combination of power surgical tools, pulse lavage, and ventilations systems can potentially produce viral load into the aerosol that may linger everywhere within the OR, forming a major source of infection for several hours or many days [2]. Pathogens can survive within particles 
smaller than $5 \mu$ in size that are suspended in the air and can propagate infection when inhaled. In such OR conditions, the spine surgeons need full personal protection $[15,30]$. Minimizing the number of staff members in the ORs is essential. It has been suggested that a dedicated team of surgeons, paramedical, and nursing staff be responsible for reviewing and operating on the suspected or definitive cases of COVID and be kept away from the rest of the department to minimize cross infections. Alternate teams may need to be created in situations of heavy caseloads or to relieve a team that has already been exposed to a suspected or infected patient [15].

\section{Special principles of intraoperative management during spine surgeries}

The following are some of the major principles to be applied during the surgery [5]: (1) The prone position is usually preferred to reduce viral transmission via aerosolized droplets. (2) It is necessary to reduce use of electrotomes and high-speed burr or drills, to ensure good intraoperative hemostasis, and to use cautiously suction devices to reduce any aerosol diffusion. (3) Surgeries must be performed gently and carefully to obviate body fluid spatter or injury by sharp instruments. (4) During surgery, nobody is allowed to enter or leave the OR, except under certain special circumstances.

\section{Postoperative care of patients undergoing spine surgery}

All patients should wear surgical masks in the postoperative period. The practice of early discharge from the hospital should be advocated, wherever feasible. All spine surgeons should promote early, aggressive rehabilitation postoperatively; and chest CT and SARS-CoV-2 nucleic acid assessment should be obtained on postoperative day 3 (for all patients undergoing surgeries and requiring hospital stay $>3$ days). After ruling out COVID-19, patients may be discharged after their condition stabilizes. However, patients with suspected or confirmed COVID during the hospital stay should be transferred to the isolation ward and managed appropriately by the medical team. The postoperative follow-up visits should be kept to a minimum and preferably carried out remotely over teleconferencing [31].

\section{Conclusions}

COVID-19 has presented a major challenge to specialty medical practices since the beginning of 2020. As discussed in this article, the field of spine surgery has already undergone a "sea of changes" during the previous few weeks; with an increase in our understanding continues to grow, still more modifications may be anticipated. A major consequence of COVID-19 crisis has been the economic strain on the state, hospitals, and surgeons. Moreover, with the subsiding of the crisis, there will be a huge strain on the health care facilities and health care providers to accommodate all patients who have had their surgeries postponed during the crisis. Such strains will be felt more seriously in the developing and under-developed economies. The apprehension of a "second wave" of infection also needs to be remembered. The need for some well thought out, timely strategies to handle such anticipated pressures in the future cannot be understated. We will need to deal with the after-effects of this pandemic for several years.

\section{Conflict of Interest}

No potential conflict of interest relevant to this article was reported.

\section{References}

1. Donnally CJ 3rd, Shenoy K, Vaccaro AR, Schroeder GD, Kepler CK. Triaging spine surgery in the COVID-19 era. Clin Spine Surg 2020;33:129-30.

2. Mavrogenis AF, Quaile A, Scarlat MM. The virus crisis affects Orthopaedic surgery and scientific activities worldwide. Int Orthop 2020;44:813-7.

3. Zhu N, Zhang D, Wang W, et al. A novel coronavirus from patients with pneumonia in China, 2019. N Engl J Med 2020;382:727-33.

4. Wrapp D, Wang N, Corbett KS, et al. Cryo-EM structure of the 2019-nCoV spike in the prefusion conformation. Science 2020;367:1260-3.

5. Zou J, Yu H, Song D, Niu J, Yang H. Advice on standardized diagnosis and treatment for spinal diseases during the coronavirus disease 2019 pandemic. Asian Spine J 2020;14:258-63.

6. Richez C, Lazaro E, Lemoine M, Truchetet ME, Schaeverbeke T. Implications of COVID-19 for the 
management of patients with inflammatory rheumatic diseases. Joint Bone Spine 2020;87:187-9.

7. Guan WJ, Ni ZY, Hu Y, et al. Clinical characteristics of coronavirus disease 2019 in China. N Engl J Med 2020;382:1708-20.

8. Lauer SA, Grantz KH, Bi Q, et al. The incubation period of coronavirus disease 2019 (COVID-19) from publicly reported confirmed cases: estimation and application. Ann Intern Med 2020;172:577-82.

9. Wang D, Hu B, Hu C, et al. Clinical characteristics of 138 hospitalized patients with 2019 novel coronavirus-infected pneumonia in Wuhan, China. JAMA 2020;323:1061-9.

10. Mo P, Xing Y, Xiao Y, et al. Clinical characteristics of refractory COVID-19 pneumonia in Wuhan, China. Clin Infect Dis 2020:ciaa270.

11. Chen N, Zhou M, Dong X, et al. Epidemiological and clinical characteristics of 99 cases of 2019 novel coronavirus pneumonia in Wuhan, China: a descriptive study. Lancet 2020;395:507-13.

12. Huang C, Wang Y, Li X, et al. Clinical features of patients infected with 2019 novel coronavirus in $\mathrm{Wu}$ han, China. Lancet 2020;395:497-506.

13. World Health Organization. Coronavirus disease (COVID-19) pandemic [Internet]. Geneva: World Health Organization; 2020 [cited 2020 Apr 30]. Available from: https://www.who.int/emergencies/ diseases/novel-coronavirus-2019.

14. Yang X, Yu Y, Xu J, et al. Clinical course and outcomes of critically ill patients with SARS-CoV-2 pneumonia in Wuhan, China: a single-centered, retrospective, observational study. Lancet Respir Med 2020;8:47581.

15. Jain VK, Vaishya R. COVID-19 and orthopaedic surgeons: the Indian scenario. Trop Doct 2020;50:10810.

16. Wang X, Pan Z, Cheng Z. Association between 2019$\mathrm{nCoV}$ transmission and $\mathrm{N} 95$ respirator use. J Hosp Infect 2020;105:104-5.

17. Jin YH, Cai L, Cheng ZS, et al. A rapid advice guideline for the diagnosis and treatment of 2019 novel coronavirus (2019-nCoV) infected pneumonia (standard version). Mil Med Res 2020;7:4.

18. Pere H, Podglajen I, Wack M, et al. Nasal swab sampling for SARS-CoV-2: a convenient alternative in times of nasopharyngeal swab shortage. J Clin Microbiol 2020;58:e00721-20.
19. Kim H, Hong H, Yoon SH. Diagnostic performance of CT and reverse transcriptase-polymerase chain reaction for coronavirus disease 2019: a meta-analysis. Radiology 2020:201343.

20. World Health Organization. Laboratory testing for coronavirus disease 2019 (COVID-19) in suspected human cases: interim guidance, 2 March 2020 [Internet]. Geneva: World Health Organization; 2020 [cited 2020 May 2]. Available from: https://apps.who.int/ iris/handle/10665/331329.

21. Fraser JF, Arthur AS, Chen M, et al. Society of NeuroInterventional Surgery recommendations for the care of emergent neurointerventional patients in the setting of COVID-19. J Neurointerv Surg 2020;12:539-41.

22. Greenland JR, Michelow MD, Wang L, London MJ. COVID-19 infection: implications for perioperative and critical care physicians. Anesthesiology 2020;132:1346-61.

23. National Centre for Disease Control. Discharge policy of 2019-nCoV case [Internet]. New Delhi: National Centre for Disease Control; 2020 [cited 2020 May 3]. Available from: https://ncdc.gov.in/WriteReadData/1892s/23914145951584779119.pdf.

24. American Hospital Association. Safely resuming elective surgery as COVID-19 curve flattens: ACS, ASA, AORN and AHA develop roadmap for readiness [Internet]. American Hospital Association; 2020 [cited 2020 May 1]. Chicago (IL): American from: https://www.aha.org/press-releases/2020-04-17safely-resuming-elective-surgery-covid-19-curveflattens-acs-asa-aorn-and.

25. American College of Surgeons; American Society of Anesthesiologists; Association of Perioperative Registered Nurses; American Hospital Association. Joint statement: roadmap for resuming elective surgery after COVID-19 pandemic [Internet]. Chicago (IL): American College of Surgeons; 2020 [cited 2020 May 1]. Available from: https://www.facs.org/covid-19/ clinical-guidance/roadmap-elective-surgery.

26. Sornsa-Ard T, Niramitsantiphong A, Liawrungrueang W. Management of traumatic spinal fracture in the coronavirus disease 2019 situation. Asian Spine J. 2020 Apr 24 [Epub]. https://doi.org/10.31616/ asj.2019.0183.

27. Rossi SL, Bortolosso H, Silva R, de Mello JMM, Costella MF. Evaluation of the efficacy of personal 
protective equipment against occupational exposure to cold. Rev Bras Med Trab 2020;17:136-44.

28. Wynants L, Van Calster B, Bonten MMJ, et al. Prediction models for diagnosis and prognosis of covid-19 infection: systematic review and critical appraisal: version 2. BMJ 2020;369:m1328.

29. Ghogawala Z, Kurpad S, Falavigna A, et al. Editorial. COVID-19 and spinal surgery. J Neurosurg Spine 2020:1-3.
30. Wong KC, Leung KS. Transmission and prevention of occupational infections in orthopaedic surgeons. J Bone Joint Surg Am 2004;86:1065-76.

31. Chang Liang Z, Wang W, Murphy D, Po Hui JH. Novel coronavirus and orthopaedic surgery: early experiences from Singapore. J Bone Joint Surg Am 2020:e000236. 